\title{
La Gestión Administrativa De Programas De Educación A Distancia Con Mediación Virtual En Universidades De Colombia
}

\author{
Mercedes Rodríguez Camargo, MA. \\ Universidad Santo Tomás, Colombia \\ Tomás Solá-Martínez, PhD. \\ Universidad de Granada, España \\ Eduardo Chaves-Barboza, PhD. \\ Universidad Nacional de Costa Rica, Costa Rica
}

doi: 10.19044/esj.2016.v12n34p90 URL:http://dx.doi.org/10.19044/esj.2016.v12n34p90

\begin{abstract}
This paper seeks to analyse the students' perception (population; 1997; sample size: 252) in three Colombian universities regarding the administrative management of the virtual distance-learning programs in which they participate. In order to reach this objective, twelve items with a four-point Likert scale were measured regarding the administrative management. In regard to the data the following tools are used: a descriptive analysis and inferential statistics; the Pearson correlation coefficient; an analysis of variance using Wilcoxon and Kruskal-Wallis tests; and a KaiserMeyer-Olkin multivariate factorial analysis with Varimax rotation.The results reveal that the programs have an adequate administrative management in the process of induction, training, extension, and support to the academic work. From the students' perspective, the administrative management is improved if the right technological infrastructure is promoted with the purpose of allowing the access to administrative services and to research.
\end{abstract}

Keywords: Teacher training, information and communication technologies, teaching and learning strategies, higher education

\section{Resumen}

El artículo tiene como objetivo analizar las percepciones que tienen los estudiantes (población 1997, muestra 252) de tres universidades colombianas sobre la gestión administrativa de programas de educación a distancia con mediación virtual, en los cuales están realizando sus estudios. Para lograr este objetivo se aplica un cuestionario tipo escala Likert con doce 
variables referidas a la gestión administrativa y cuatro niveles de frecuencia. Sobre los datos se realizan análisis de estadística descriptiva e inferencial, se calculan coeficientes de correlación de Pearson, se efectúan análisis de varianza mediante pruebas de Wilcoxon y tests Kruskal-Wallis, y se hacen análisis factoriales multivariados Kaiser-Meyer-Olkin con rotación Varimax. Como resultados se obtiene que los programas de estudio cuentan con una adecuada gestión administrativa en los procesos de inducción, capacitación, divulgación y apoyo de la labor académica. Desde la perspectiva de los estudiantes se mejora la gestión administrativa si se fomenta una adecuada infraestructura tecnológica que permita acceder a los servicios administrativos y a la investigación.

Palabras clave: Formación del profesorado, tecnologías de información y comunicación, estrategias de enseñanza aprendizaje, educación superior

\section{Introducción}

En el ámbito educativo mundial se observa un proceso para lograr que las tecnologías de la información y la comunicación (TIC) sean accesibles a todas las poblaciones humanas, principalmente a través de Internet. En el caso de Colombia este proceso ha alcanzado auge hasta la presente década (OCDE, 2016), para esto se ha comenzado a incorporar personal capacitado en informática, pero con escasa formación pedagógica, principalmente para que capacite en el manejo de herramientas ofimáticas (Bitrán, Benavente y Maggi, 2011). La educación a distancia no es la excepción. Se menciona esta modalidad porque en Colombia, la educación a distancia tienen una importancia notable (Contreras, Leal y Salazar, 2001) debido, principalmente, a que en las historia educativa colombiana esta modalidad generó una dinámica de aumento en la calidad para el sistema de educación superior. Y aunque la demanda es $87 \%$ menor que la presencial (Arboleda y Rama, 2013), las políticas nacionales incorporan las exigencias de la educación mundial tomando como referencia la educación a distancia (Gómez y Sánchez, 2013).

Si, además, consideramos que en la educación a distancia colombiana la presencia de las TIC es uno de los elementos que más le han dado relevancia y presencia social, se hace particularmente necesario el análisis del apoyo que las herramientas virtuales tienen en estos procesos educativos.

Cabe aclarar que la modalidad de educación a distancia se estableció para continuar y culminar lo procesos educativos de una población adulta, con la posibilidad de continuar trabajando. Esto se veía como novedad hace cuarenta y cinco años, sus inicios fueron artesanales y eclécticos al unir varios elementos como los encuentros tutoriales, la utilización de libros, los procesos de matrículas adaptados, entre otros actos (Ruíz, Gómez, Lara y 
Mestre, 2013). Esta modalidad se concebía como una alternativa para el acercamiento entre la educación formal y el ciudadano que se encontraba en una zona alejada de las urbes, las políticas no habían contemplado aún el avance de la tecnología (Morales, Trujillo y Raso, 2015).

La problemática que surge de estos cuestionamientos, se planteó el distinguir las tensiones que se presentan entre la normatividad expuesta por el Ministerio de Educación Nacional, lo establecido en las universidades de educación a distancia y lo que reciben como educación los estudiantes, esta relación tripartita permitirá señalar la calidad de la educación a distancia con mediación virtual en Colombia, para ello se retomarán algunos elementos que generen tensión como son la autonomía universitaria señalada por la normatividad Colombiana y las propuestas de orden internacional con referencia a la modalidad.

Para el abordaje y comprensión del tema se traza como objetivo general, analizar la gestión educativa de la Educación a Distancia en los escenarios de la formación superior en Colombia señalando los aciertos y desaciertos de la misma frente a las exigencias que señalan las políticas internacionales. En el proceso de desarrollo de la investigación con miras a obtener la meta se perfilan los objetivos específicos de Identificar, contextualizar y comparar la educación a distancia, su calidad, interacción con el medio virtual e incidencias en la educación superior en Colombia.

\section{La educación a distancia con mediación virtual}

La educación a distancia con mediación virtual empieza a desplegarse en 1998 (Facundo, 2005), y va dirigida a mejorar la calidad de vida de las poblaciones y a avanzar en la construcción de una sociedad democrática, vista desde la participación en la información y la toma de decisiones responsables (Areth, Castro y Rodríguez; 2014). Actualmente la educación a distancia colombiana no solo establece una excelente cobertura nacional, sino que sus extensiones son internacionales.

En efecto, las instituciones colombianas que han sido pioneras en la educación a distancia han desarrollado sus programas dando énfasis a la gestión administrativa y al apoyo de tecnologías virtuales adaptados a la modalidad (Morantes y Acuña, 2013). Esto se refleja en sus proyecciones institucionales y en los marcos de acreditación nacional para estos programas educativos (Arboleda y Rama, 2013). Por lo tanto, en el caso concreto de Colombia es muy pertinente relacionar investigativamente la gestión administrativa y el apoyo de las tecnologías virtuales, como variables para el análisis de los programas de educación a distancia.

\section{Metodología de investigación}

La metodología a realizar en esta investigación se centra en el 
paradigma Mixto entre lo cualitativo- cuantitativo y un énfasis hermenéutico.

\section{Poblaciones y muestras}

La población en estudio está compuesta por los 1997 estudiantes de pregrado de 3 universidades colombianas que estudian bajo la modalidad a distancia con mediación virtual. La muestra de 252 estudiantes ha sido seleccionada mediante un muestreo aleatorio simple, lo que permite el cálculo de intervalos de confianza (IC) con una significancia del 95\% y un

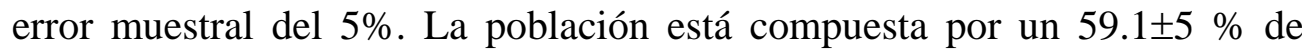
mujeres y tiene un promedio de $31.53 \pm 1.58$ años de edad.

Las tres universidades, dos privadas y una pública, tienen más de 40 años en la formación bajo la modalidad de educación a distancia y poseen reconocimiento a nivel nacional.

\section{El cuestionario y sus variables}

El cuestionario aplicado tiene una introducción que presenta a los encargados de la investigación, el objetivo del estudio, un compromiso con la confidencialidad de los participantes y una solicitud de colaboración.

Luego de unas instrucciones sobre cómo responder, el cuestionario tiene preguntas iniciales que tratan sobre la edad, el sexo, nombre de la institución educativa y si es pública o privada.

Posteriormente, se presentan las preguntas principales, éstas miden la percepción de los sujetos sobre la gestión administrativa del programa de educación a distancia con mediación virtual en la cual estudia. Está parte está conformada por doce ítems tipo escala Likert con cuatro niveles: nunca, pocas veces, algunas veces, casi siempre, siempre.

\section{Pruebas estadísticas e hipótesis}

Para analizar los datos obtenidos con el cuestionario se aplican técnicas de estadísticas descriptivas e inferencial, análisis bivariados de correlación, pruebas de variabilidad y análisis factoriales multivariados.

Las estadísticas descriptivas e inferenciales estudia las frecuencias relativas en los distintos niveles de las variables, la inferencia estadística se realiza con un error estadístico del 5\% y un nivel de confianza del 95\%.

Los análisis de correlación se realizan mediante el cálculo del coeficiente $\rho$ de Pearson al 95\% del nivel de confianza. En estos casos, para cada par de variables se plantean las hipótesis usuales de correlación lineal, esto es la hipótesis nula plantea que la correlación es cero mientras que la alternativa asume que es diferente de cero.

Los análisis de varianza se aplican para establecer si existen diferencias significativas entre distintos niveles de las variables en estudio. Estos niveles son respuestas de los estudiantes en distintas variables de la 
gestión administrativa (dos niveles) para comparar estadísticos como promedios y desviaciones estándar, o bien grupos que eligieron alguna de las opciones de la escala Likert en las distintas variables (cinco niveles). En los casos de comparación de estadísticos entre dos variables se aplican pruebas de rangos de muestras relacionadas Wilcoxon y en el caso de análisis de varianza entre grupos independientes para los cinco niveles Likert se aplican tests Kruskal-Wallis; en ambos casos se busca determinar la influencia de los cambios de nivel sobre la gestión administrativa. En los análisis de varianza la hipótesis nula plantea que la variable dependiente permanece igual a distintos niveles de la variable independiente mientras la hipótesis alternativa asume que la variable dependiente no permanece igual.

Finalmente, para los análisis factoriales multivariados se aplica el procedimiento Kaiser-Meyer-Olkin (KMO), con test de Barlett y rotación Varimax con normalización de Kaiser (método de extracción por componentes principales). Para el test de Barlett se utiliza como hipótesis nula que las variables no están correlacionadas (matriz de correlación igual a la identidad), la hipótesis alternativa es que el coeficiente de correlación bivariado es diferente de cero en cada caso. El análisis factorial permite reducir el conjunto de variables de la dimensión a un número menor de factores (espacio dimensional) que lo representa, lo cual facilita su interpretación.

\section{Validez y confiabilidad del cuestionario}

La congruencia de los ítems del cuestionario respecto a las dimensiones ha sido revisada por diez profesores universitarios con experiencia superior a tres (3) años en educación a distancia. Además, se hizo una aplicación piloto a 10 estudiantes y 10 profesores para evaluar la claridad de las preguntas, obteniendo todos lo ítems más del $70 \%$ de aceptación. La fiabilidad estadística del cuestionario ha sido analizada mediante el coeficiente alfa de Cronbach, alcanzando un valor de .907 en este estadístico, por lo tanto su nivel de confiabilidad es adecuada.

\section{Resultados de las pruebas estadísticas}

\section{Análisis sobre variables de gestión administrativa}

La Tabla 1 muestra, para cada variable de gestión administrativa, la frecuencia relativa, el promedio y la desviación estándar, según la percepción de los estudiantes sobre los programas de educación a distancia con mediación virtual.

Como puede verse en esta tabla, las doce variables muestran diferencias significativas entre los niveles de frecuencia, a favor del siempre y el casi siempre, según la prueba Kruskal-Wallis, $\mathrm{p}<.05$, dos colas, un nivel de confianza del 95\% y un error estándar del 5\%. Los promedios para las 
doce variables son mayores que 3 (el valor de la media).

Se debe prestar gran atención a las variables "Se encuentra la infraestructura tecnológica necesaria para el acceso a la modalidad", "Las políticas de fortalecimiento de nuevas tecnologías son divulgadas por la administración académica", "El proceso de ingreso a la modalidad refleja una adecuada organización administrativa", "Las políticas administrativas apoyan las gestiones académicas", "Las directrices administrativas son coherentes con la infraestructura tecnológica que requiere la educación a distancia” y "Las políticas de las nuevas tecnologías son apropiadas para el personal administrativo" que muestra promedios relativamente altos (4.23, 4.14, 4.12, 4.08, 4.06 y 4.04 respectivamente).

Tabla 1: Variables de gestión administrativa, según estudiantes

FRECUENCIAS RELATIVAS ${ }^{(1)}$

\begin{tabular}{|c|c|c|c|c|c|c|c|}
\hline VARIABLES & Nunca & $\begin{array}{c}\text { Casi } \\
\text { nunca }\end{array}$ & $\begin{array}{l}\text { Algunas } \\
\text { veces }\end{array}$ & $\begin{array}{l}\text { Casi } \\
\text { siempre }\end{array}$ & Siempre & $\bar{X}^{(2)}$ & $\sigma^{(3)}$ \\
\hline $\begin{array}{l}\text { Las políticas de fortalecimiento de nuevas } \\
\text { tecnologías son divulgadas por la } \\
\text { administración académica }^{(4)}\end{array}$ & 0.4 & 3.6 & 19.4 & 34.5 & 42.1 & 4,14 & 0,88 \\
\hline $\begin{array}{l}\text { Las políticas de las nuevas tecnologías son } \\
\text { apropiadas para el personal administrativo }\end{array}$ & 0.8 & 2.8 & 22.6 & 38.9 & 34.9 & 4,04 & 0,87 \\
\hline $\begin{array}{c}\text { El apoyo administrativo se observa en el plan } \\
\text { de mejoramiento }{ }^{(4)}\end{array}$ & 2.4 & 7.9 & 25.4 & 33.7 & 30.6 & 3,82 & 1,03 \\
\hline $\begin{array}{l}\text { El proceso de ingreso a la modalidad refleja } \\
\text { una adecuada organización administrativa }{ }^{(4)}\end{array}$ & 0.8 & 3.6 & 20.6 & 32.5 & 42.5 & 4,12 & 0,91 \\
\hline $\begin{array}{l}\text { Las políticas administrativas apoyan las } \\
\text { gestiones académicas }{ }^{(4)}\end{array}$ & 1.2 & 3.2 & 21.4 & 35.3 & 38.9 & 4,08 & 0,91 \\
\hline $\begin{array}{l}\text { Las directrices administrativas son coherentes } \\
\text { con la infraestructura tecnológica que } \\
\text { requiere la educación a distancia }{ }^{(4)}\end{array}$ & 2.0 & 3.6 & 17.9 & 39.7 & 36.9 & 4,06 & 0,93 \\
\hline $\begin{array}{l}\text { Se brinda capacitación e inducción para el } \\
\text { ingreso al aula virtual }{ }^{(4)}\end{array}$ & 4.8 & 9.5 & 17.1 & 26.6 & 42.1 & 3,92 & 1,18 \\
\hline $\begin{array}{c}\text { Se cuenta con herramientas web } 2.0 \text { para la } \\
\text { investigación }{ }^{(4)}\end{array}$ & 2.0 & 6.7 & 23.0 & 36.9 & 31.3 & 3,89 & 0,99 \\
\hline $\begin{array}{l}\text { Hay acceso a base de datos para } \\
\text { investigación }{ }^{(4)}\end{array}$ & 4.0 & 9.1 & 23.8 & 32.1 & 31.0 & 3,77 & 1,10 \\
\hline $\begin{array}{l}\text { Las dependencias de la institución están } \\
\text { vinculadas a la plataforma }^{(4)}\end{array}$ & 2.0 & 5.6 & 20.6 & 36.9 & 34.9 & 3,97 & 0,98 \\
\hline $\begin{array}{l}\text { Se obtienen informes académicos a través del } \\
\text { correo institucional }{ }^{(4)}\end{array}$ & 7.5 & 8.7 & 23.0 & 25.8 & 34.9 & 3,72 & 1,23 \\
\hline $\begin{array}{l}\text { Se encuentra la infraestructura tecnológica } \\
\text { necesaria para el acceso a la modalidad }{ }^{(4)}\end{array}$ & 0.8 & 0.8 & 16.3 & 38.9 & 43.3 & 4,23 & 0,80 \\
\hline
\end{tabular}

N = 1997. n = 252. (1) Error muestral del 5\%. (2) $\bar{X}=$ Promedio muestral. (3) $\sigma=$ Desviación estándar muestral.

(4) Variable con diferencias estadísticamente significativas entre niveles de respuesta en escala Likert, según prueba Kruskal-Wallis, $\mathrm{p}<.05$, dos colas. 
Aún las variables "Se obtienen informes académicos a través del correo institucional” y "Hay acceso a base de datos para investigación”, que muestran los promedios más bajos (3.72 y 3.77 respectivamente), tienen diferencias significativas con la media, según la prueba Kruskal-Wallis, $\mathrm{p}<.05$, dos colas, un nivel de confianza del 95\% y un error estándar del 5\%.

Figura 1: Comparación entre variables de gestión administrativa

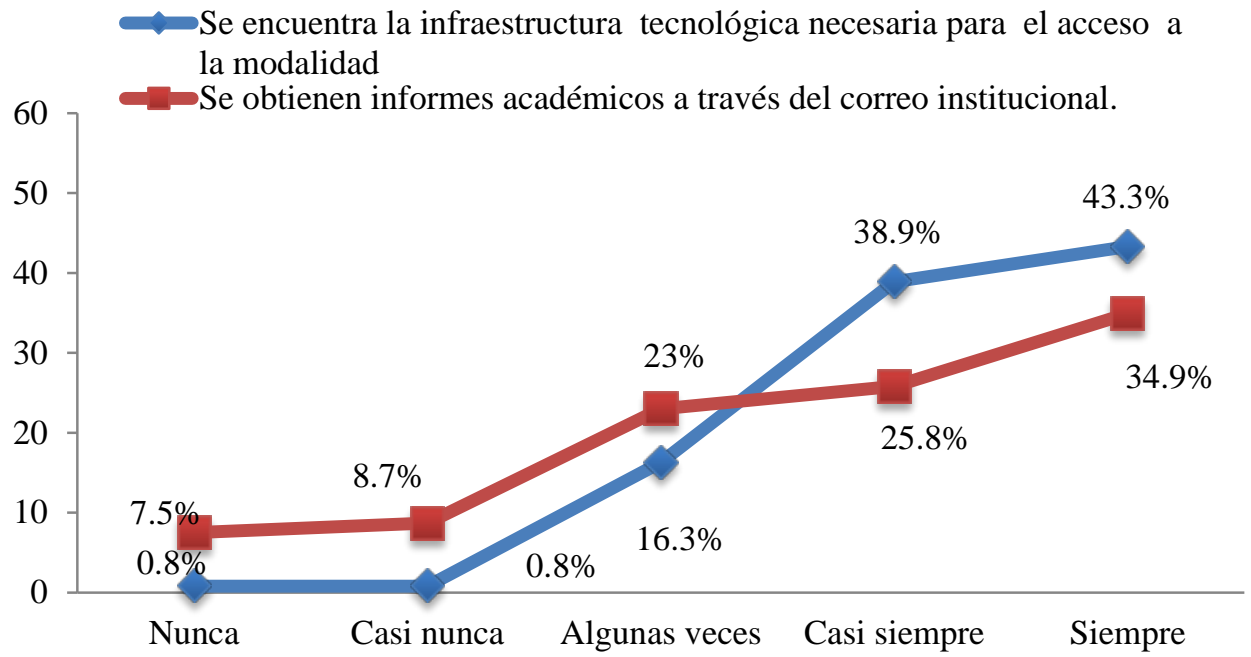

Esto significa que una mayoría muy significativa de la población en estudio considera que las doce variables se observan siempre o casi siempre en los programas de estudio a distancia que participaron en el estudio.

En la Figura 1 se comparan la variable de gestión administrativa que muestra el mayor promedio y la variable que presenta el menor promedio, y ambas muestran frecuencias relativas elevadas en las categorías de siempre y casi siempre, y frecuencias relativas bajas en las categorías de nunca y casi nunca.

Por otra parte, según se observa en la Tabla 1, en las variables "Se obtienen informes académicos a través del correo institucional", "Se brinda capacitación e inducción para el ingreso al aula virtual" y "Hay acceso a base de datos para investigación" se observa una mayor dispersión de las respuestas que en las variables "Se encuentra la infraestructura tecnológica necesaria para el acceso a la modalidad", "Las políticas de las nuevas tecnologías son apropiadas para el personal administrativo" y "Las políticas de fortalecimiento de nuevas tecnologías son divulgadas por la administración académica". De hecho, existen diferencias estadísticamente significativas entre las desviaciones estándar de estas tres últimas variables y las primeras tres, según prueba Wilcoxon, $\mathrm{p}<.05$, dos colas, un nivel de confianza del 95\% y error estándar del 5\%. 


\section{Relación entre variables de gestión administrativa}

En la Tabla 2 se relacionan las variables de gestión administrativa de los programas en estudio. Para esto se muestran solo los coeficientes de correlación estadísticamente significativos para su comparación.

Las variables "Las políticas de fortalecimiento de nuevas tecnologías son divulgadas por la administración académica", "Las políticas de las nuevas tecnologías son apropiadas para el personal administrativo", "El apoyo administrativo se observa en el plan de mejoramiento", "El proceso de ingreso a la modalidad refleja una organización administrativa", "Las políticas administrativas apoyan las gestiones académicas" y "Las directrices administrativas son coherentes con la infraestructura tecnológica que requiere la educación a distancia" forman un subconjunto de variables fuerte y positivamente correlacionadas entre sí, con $.513 \leq \rho \leq .703$.

Asimismo, tienen elevadas y positivas correlaciones positivas entre sí las variables "Se cuenta con herramientas web 2.0 para la investigación", "Hay acceso a base de datos para investigación" y "Se encuentra la infraestructura tecnológica necesaria para el acceso a la modalidad”. Estas variables forman un segundo subconjunto de variables correlacionadas con $.506 \leq \rho \leq .551$.

Nótese que la variable "Las políticas administrativas apoyan las gestiones académicas” pertenece al primer subconjunto, y además, está significativamente relacionada con la variables "Se cuenta con herramientas web 2.0 para la investigación”, que pertenece al segundo subconjunto.

Tabla 2: Correlación entre variables de gestión administrativa

\begin{tabular}{|c|c|c|c|c|c|c|c|c|}
\hline & \multirow[b]{2}{*}{ VARIABLES } & \multicolumn{7}{|c|}{ COEFICIENTE $\rho$ DE PEARSON } \\
\hline & & 1 & 2 & 3 & 4 & 5 & 6 & 7 \\
\hline 1 & $\begin{array}{c}\text { Las políticas de fortalecimiento de nuevas } \\
\text { tecnologías son divulgadas por la } \\
\text { administración académica }\end{array}$ & - & & & & & & \\
\hline 2 & $\begin{array}{l}\text { Las políticas de las nuevas tecnologías son } \\
\text { apropiadas para el personal administrativo }\end{array}$ & .703 & & & & & & \\
\hline 3 & $\begin{array}{l}\text { El apoyo administrativo se observa en el } \\
\text { plan de mejoramiento }\end{array}$ & .620 & .612 & & & & & \\
\hline 4 & $\begin{array}{c}\text { El proceso de ingreso a la modalidad refleja } \\
\text { una organización administrativa }\end{array}$ & .513 & .529 & .637 & & & & \\
\hline 5 & $\begin{array}{c}\text { Las políticas administrativas apoyan las } \\
\text { gestiones académicas }\end{array}$ & .595 & .586 & .661 & .595 & & & \\
\hline 6 & $\begin{array}{c}\text { Se cuenta con herramientas web } 2.0 \text { para la } \\
\text { investigación. }\end{array}$ & - & - & - & - & .506 & & \\
\hline 7 & $\begin{array}{c}\text { Hay acceso a base de datos para } \\
\text { investigación }\end{array}$ & - & - & - & - & - & .551 & \\
\hline
\end{tabular}


COEFICIENTE $\rho$ DE PEARSON

VARIABLES

1

2

3

$45 \quad 6$

8 Las directrices administrativas son

coherentes con la infraestructura

tecnológica que requiere la educación a

$\begin{array}{llll}.557 \quad 620 & .658 & .609 & .673\end{array}$

distancia

9 Se encuentra la infraestructura tecnológica

necesaria para el acceso a la modalidad

$\mathrm{N}=$ 1997. $\mathrm{n}=2$ 252. En todos los casos $\mathrm{p}<.01$, dos colas, error muestral del $5 \%$

Variabilidad de la gestión administrativa explicado por las variables en estudio y factores derivados

El análisis factorial multivariado permite determinar dos factores que explican el 60.84\% de la variabilidad de la gestión administrativa, con KMO $=.926, \chi^{2}=1539.04$, Sig. $<.0001$, con error muestral del 5\%. Todas las variables tienen una fuerte relación con los factores retenidos. El primer factor explica el $51.46 \%$ y el segundo factor el $9.38 \%$.

La Tabla 3 muestra los porcentajes de varianza de la gestión administrativa que pueden ser explicados por cada factor y cada variable, para la población de estudiantes. Según la Tabla 3, el factor con mayor porcentaje de poder explicativo sobre la gestión administrativa es el primero, que explican un $51.46 \%$ de la varianza.

Tabla 3: Porcentaje de la gestión administrativa explicado por las variables en estudio y factores derivados de ellas, según estudiantes

\begin{tabular}{ccccc}
\hline Variable & Factor & $\begin{array}{c}\text { \% de variabilidad que } \\
\text { explica el factor }\end{array}$ & $\begin{array}{c}\text { Coeficiente de } \\
\text { correlación de la } \\
\text { variable con su factor }\end{array}$ & $\begin{array}{c}\text { \% de variabilidad que } \\
\text { explica la variable }\end{array}$ \\
\hline $\begin{array}{c}\text { Las políticas de fortalecimiento de } \\
\text { nuevas tecnologías son divulgadas } \\
\text { por la administración académica } \\
\quad \text { Las políticas de las nuevas } \\
\text { tecnologías son apropiadas para el } \\
\text { personal administrativo }\end{array}$ & 1 & 51.46 & .76 & 39.11 \\
$\begin{array}{c}\text { El apoyo administrativo se observa } \\
\text { en el plan de mejoramiento }\end{array}$ & 1 & 51.46 & .84 & 43.23 \\
$\begin{array}{c}\text { El proceso de ingreso a la modalidad } \\
\text { refleja una adecuada organización } \\
\text { administrativa }\end{array}$ & 1 & 51.46 & .77 & 39.62 \\
$\begin{array}{c}\text { Las políticas administrativas apoyan } \\
\text { las gestiones académicas }\end{array}$ & 1 & 51.46 & .70 & 36.02 \\
$\begin{array}{c}\text { Las directrices administrativas son } \\
\text { coherentes con la infraestructura } \\
\text { tecnológica que requiere la } \\
\text { educación a distancia }\end{array}$ & 1 & 51.46 & .74 & 38.68 \\
\end{tabular}




\begin{tabular}{|c|c|c|c|c|}
\hline Variable & Factor & $\begin{array}{l}\text { \% de variabilidad que } \\
\text { explica el factor }\end{array}$ & $\begin{array}{c}\text { Coeficiente de } \\
\text { correlación de la } \\
\text { variable con su factor }\end{array}$ & $\begin{array}{l}\text { \% de variabilidad que } \\
\text { explica la variable }\end{array}$ \\
\hline $\begin{array}{l}\text { Se brinda capacitación e inducción } \\
\text { para el ingreso al aula virtual }\end{array}$ & 2 & 9.38 & .66 & 6.19 \\
\hline $\begin{array}{c}\text { Se cuenta con herramientas web } 2.0 \\
\text { para la investigación. }\end{array}$ & 2 & 9.38 & .75 & 7.04 \\
\hline $\begin{array}{c}\text { Hay acceso a base de datos para } \\
\text { investigación }\end{array}$ & 2 & 9.38 & .72 & 6.75 \\
\hline $\begin{array}{l}\text { Las dependencias de la institución } \\
\text { están vinculadas a la plataforma }\end{array}$ & 2 & 9.38 & .56 & 5.25 \\
\hline $\begin{array}{l}\text { Se obtienen informes académicos a } \\
\text { través del correo institucional. }\end{array}$ & 2 & 9.38 & .60 & 5.63 \\
\hline $\begin{array}{l}\text { Se encuentra la infraestructura } \\
\text { tecnológica necesaria para el acceso } \\
\text { a la modalidad. }\end{array}$ & 2 & 9.38 & .73 & 6.85 \\
\hline
\end{tabular}

Método de extracción por componentes principales. Método de rotación Varimax con normalización de Kaiser. Error muestral del 5\%. Convergencia lograda con 3 rotaciones. $\mathrm{N}=1997, \mathrm{n}=256, \mathrm{KMO}=$ $.926, \chi^{2}=1536.04$, Sig. $<.0001$

Gracias al método de rotación Varimax, se ha logrado que el primer factor estén compuesto por las variables con mayores saturaciones y que estén muy relacionadas entre sí, por lo tanto son las que mayor poder explicativo tienen sobre el la gestión administrativa.

Como puede observarse en la Tabla 3, el primer factor está compuesto por las variables "Las políticas de fortalecimiento de nuevas tecnologías son divulgadas por la administración académica", "Las políticas de las nuevas tecnologías son apropiadas para el personal administrativo", "El apoyo administrativo se observa en el plan de mejoramiento", "El proceso de ingreso a la modalidad refleja una adecuada organización administrativa", "Las políticas administrativas apoyan las gestiones académicas" y "Las directrices administrativas son coherentes con la infraestructura tecnológica que requiere la educación a distancia". Estas son las variables que tienen más poder explicativo sobre la gestión administrativa, según la percepción de los estudiantes, e influyen significativamente sobre la gestión administrativa explicando este fenómeno con porcentajes entre un $36.02 \%$ y un $43.23 \%$.

\section{Discusión}

La población de estudiantes percibe que sus programas de educación a distancia con mediación virtual se caracterizan porque tienen la infraestructura tecnológica necesaria para el acceso a la modalidad. Los programas cuentan con herramientas web 2.0 y acceso a base de datos para 
la investigación. Asimismo, las dependencias de la institución están vinculadas a la plataforma, lo que permite que los estudiantes puedan acceder a los servicios administrativos en línea, por ejemplo, pueden obtener informes académicos a través del correo institucional. Los estudiantes señalan que la presencia de infraestructura necesaria para el acceso a la modalidad está muy relacionado con la existencia de herramientas y el acceso a bases de datos necesarios para la investigación, esto subraya la importancia que los estudiantes otorgan a la investigación en esta modalidad de programas.

Los estudiantes opinan que las políticas administrativas apoyan las gestiones académicas y el plan de mejoramiento tecnológico, las directrices administrativas son coherentes con la infraestructura tecnológica que requiere la educación a distancia y las políticas de las nuevas tecnologías son apropiadas para el personal administrativo. Los estudiantes perciben que todos estos elementos de gestión administrativa están muy relacionados, lo que significa que el encontrar políticas y directrices de mejoramiento tecnológico adecuado y coherente para el personal de gestión administrativa es importante para lograr su apoyo en la gestión académica.

Los estudiantes perciben que los programas cuentan con una adecuada organización administrativa de acuerdo a la modalidad a distancia con mediación virtual, esto es percibido desde el ingreso al programa, en este sentido, se brinda capacitación e inducción para el ingreso en el aula virtual. En general, las políticas de fortalecimiento de nuevas tecnologías son debidamente divulgadas y apoyadas por la administración académica. Las pruebas señalan que estas características están muy correlacionadas entre sí, lo que resalta la importancia de adecuados procesos de capacitación, inducción y divulgación de los procesos de mejoramiento tecnológicos, esto para que la modalidad a distancia con mediación virtual cuente con una adecuada organización administrativa.

El análisis factorial señala que la adecuación de las políticas para el personal administrativo es una variable que influye positiva y decisivamente sobre la gestión administrativa, de igual modo es influye que las directrices administrativas sean coherentes con la infraestructura tecnológica de la educación a distancia. También, influyen que el personal administrativo tenga participación en el plan de mejoramiento, en el proceso de ingreso a la modalidad, en la divulgación de las políticas de fortalecimiento y en la gestión académica. Por estos resultados del análisis factorial, se recomienda trabajar en la coherencia y adecuación de las políticas y directrices de implementación tecnológica en los programas de educación a distancia. También se recomienda, en los programas de educación a distancia, realizar los procesos de divulgación, capacitación y aplicación de las tecnologías virtuales, contando con una amplia participación del personal encargado de 
la gestión administrativa del programa.

\section{Conclusión}

El estudio de variables de gestión administrativa permite ofrecer las siguientes conclusiones y recomendaciones:

1. En un programa de educación a distancia con apoyo virtual, los estudiantes perciben como características de una buena gestión administrativa el contar con una adecuada infraestructura tecnológica que permita acceder a los servicios administrativos y a la investigación. Para mejorar la gestión administrativa de un programa de esta naturaleza se recomienda fortalecer estos elementos.

2. Se recomienda fomentar la coherencia y la adecuación de las políticas y directrices para la gestión administrativa, según la modalidad del programa de educación a distancia con apoyo de tecnologías virtuales. Esto es percibido por los estudiantes como características de una buena gestión administrativa.

3. Los estudiantes perciben que sus programas de estudio cuentan con una adecuada organización administrativa de acuerdo a la modalidad a distancia con mediación virtual, esto es percibido tanto en el proceso de ingreso a al programa como en los procesos de inducción, capacitación, divulgación y apoyo de la gestión académica.

4. Para mejorar la gestión administrativa, se recomienda darle amplia participación al personal encargado de la gestión administrativa en todos los procesos de capacitación, de divulgación y de aplicación de proyectos de mejoramiento de los programas, en especial si involucran tecnologías virtuales.

\section{References:}

5. Areth, J.; Castro, J. y Rodriguez, H. (2014). La educación virtual en Colombia: exposición de Modelos de deserción. Institución Universitaria Politécnico Gran Colombia. Recuperado de http://repository.poligran.edu.co/bitstream/10823/570/1/2014.06.24.A RTICULO\%20EDUCACION\%20VIRTUAL.pdf

6. Arboleda, N. y Rama, C. (2013).La Educación Superior a distancia y virtual en Colombia: nuevas realidades. Bogotá: ACESAD. Recuperado de http:/virtualeduca.org/documentos/observatorio/la_educacion_superi or_a_distancia_y_virtual_en_colombia_nuevas_realidades.pdf

7. Bitrán, E; Benavente, J. y Maggi, C. (2011). Informe de consultoría. Bases para una estrategia de innovación y competitividad para Colombia. Centro de productividad. Universidad Adolfo IbáñezChile. (pp 1-36). Recuperado de http://www.urosario.edu.co/ICTPI- 
11/Documentos/Estrategia-innovacion-Colombia-VDEF-AbstractBitra/

8. Contreras, M., Leal, J. y Salazar R. (2001). Educación a distancia. Bogotá: Ediciones hispanoamericanas, p 234

9. Facundo, Á. (2005). La educación superior a distancia/virtual en Colombia. Recuperado de: http://portales.puj.edu.co/didactica/PDF/Tecnologia/Educacionvirtual enColombia.pdf (Consulta mayo de 2015)

10. Gómez, H. y Sánchez, V.(2013) "Indicadores cualitativos para la mediación de la calidad en educación”. Revista Edu.Educ, (Vol. 16 No. 1, pp. 9-24). Universidad de la Sabana. Facultad de Educación.

11. Morales, M., Trujillo, J.M. y Raso, F. (2015). Percepciones acerca de la integración de las TIC en el proceso de enseñanza-aprendizaje de la universidad. Pixel-Bit. Revista de Medios y Educación (No. 46. Enero 2015, pp. 103-117). Universidad de Granada. Facultad de ciencias y educación. Recuperado de: http://acdc.sav.us.es/pixelbit/images/stories/p46/07.pdf. Consultado en Mayo 10 de 2016.

12. Morantes, A. y Acuña, G. (2013). "Propuesta de modelo de gestión para Educación Superior a Distancia: una aproximación”. Zona próxima. Revista del Instituto de Estudios en Educación Universidad del Norte. (18, pp. 72-92). Recuperado de: http://www.redalyc.org/articulo.oa?id=85328617007.

13. OCDE (2016). Revisión de políticas nacionales de educación: La educación en Colombia. Recuperado en http://www.mineducacion.gov.co/1759/articles356787_recurso_1.pdf

14. Ruíz, E.; Gómez, J.; Lara, J. y Mestre, G. (2013). Modelos de educación a Distancia. Universidad Tecnológica de Bolívar. Recuperado de: http://www.unitecnologica.edu.co/educacionadistancia/sites/default/fi les/Investigaci\%C3\%B3n\%20Documento\%20Creaci\%C3\%B3n\%20CEaD\%20UTB.pdf 